\title{
ÉTALE $K$-THEORY AND ARITHMETIC
}

\author{
BY WILLIAM G. DWYER AND ERIC M. FRIEDLANDER ${ }^{1}$
}

The purpose of this note is to announce some new results about the algebraic $K$-theory of rings of integers in global fields.

THEOREM 1. Let $O$ denote the ring of integers in a number field $K$ (i.e. a finite extension field of the rational numbers $\mathbf{Q})$ and let $l$ be an odd prime. Then there are natural surjective maps

$$
\mathrm{ch}_{i, k}: K_{2 i-k}(O) \otimes \mathrm{Z}_{l} \rightarrow H^{k}\left(O[1 / l], \mathrm{Z}_{l}(i)\right), \quad k=1 \text { or } 2,2 i-k>1 .
$$

REMARK. The requirement that $l$ be an odd prime can be dropped if $K$ is totally imaginary.

The groups on the right of (1.1) are continuous $l$-adic étale cohomology groups. Recall that $\mathbf{Z} / l^{\nu}(1)$ denotes the sheaf of $l^{v}$ th roots of unity, $\mathbf{Z} / l^{\nu}(i)=$ $\left(\mathbf{Z} / l^{v}(1)\right)^{\otimes i}$, and $\mathbf{Z}_{l}(i)=\lim _{\leftarrow} \mathbf{Z} / l^{v}(i)$. D. Quillen has conjectured the existence of isomorphisms of type (1.1). B. Harris and G. Segal [4] have shown that (1.1) is surjective on torsion if $k=1$; C. Soule [6] in many cases proved surjectivity for $k=2$ with $i<l$.

The surjectivity of (1.1) together with A. Borel's computation of $K_{*}(0) \otimes$ Q [1] gives a new proof of the existence [7] of isomorphisms

$$
\mathrm{ch}_{i, k} \otimes \mathrm{Q}: K_{2 i-k}(\mathrm{O}) \otimes \mathrm{Q}_{l} \stackrel{\sim}{\rightarrow} H^{k}\left(\mathrm{O}[1 / l], \mathrm{Q}_{l}(i)\right) \text {. }
$$

In particular, Theorem 1 implies that $\mathrm{ch}_{i, 1}$ detects "Borel classes" in $K_{2 i-1}(0)$ (i.e. basis elements for $K_{2 i-1}(0) /$ torsion). This leads to the following corollary, which is consistent with long-standing conjectures about the algebraic $K$-theory with finite coefficients of the algebraic closure of $\mathbf{Q}$.

COROLlary 2. For any integers $i \geqslant 1$ and $v>0$ there exists a finite solvable field extension $K^{\prime}$ of $K$ with ring of integers $O^{\prime}$ such that the image of $K_{2 i-1}(0) /$ torsion in $K_{2 i-1}\left(O^{\prime}\right) /$ torsion is divisible by $l^{v}$.

Conjectures by S. Lichtenbaum [5] and work by Lichtenbaum and others relate the values of the Dedekind zeta function of $K$ at negative integers to the number of elements of finite order in the groups $H^{k}\left(O[1 / l], \mathbf{Z}_{l}(i)\right)$. For example, combining (1) with known properties of Bernoulli numbers gives the new result that $K_{134}(Z)$ contains an element of order 37.

Received by the editors November 12, 1981 and, in revised form, January 4, 1982.

1980 Mathematics Subject Classification. Primary 18F25; Secondary 12A60, 55N15.

1 Research partially supported by the N. S. F. 
We have also proved a theorem analogous to Theorem 1 in the function field case.

THEOREM 3. Let $A$ be a ring of integers in a function field of characteristic $p$ (i.e., a finite extension of $\mathbf{F}_{p}(t)$ ) and let $l$ be a prime different from $p$. Then there are natural surjective maps

$$
\operatorname{ch}_{i, k}: K_{2 i-k}(A) \rightarrow H^{k}\left(A, \mathrm{Z}_{l}(i)\right), \quad k=1 \text { or } 2,2 i-k>0 .
$$

This was first proved by C. Soule for $i<l[6]$.

$\mathrm{R}$. Thomason has recently awakened interest in a type of $K$-theory which is obtained from ordinary algebraic $K$-theory with coefficients by imposing a periodicity [8]. It is known that in many cases the periodic analogue of map 1.1 is either a split epimorphism [2] or even an isomorphism [9].

The proofs of Theorems 1 and 3 use étale $K$-theory, a twisted generalized cohomology theory on the étale homotopy type of a noetherian ring (or scheme). We extend the theory developed in [3] for varieties over an algebraically closed field to the setting of schemes over $\mathbf{Z}[1 / l]$. In particular, this gives the following.

THEOREM 4. There are natural transformations of ring-valued functions

$$
\begin{gathered}
\hat{\varphi}_{*}: K_{*}() \rightarrow \hat{K}_{*}^{\mathrm{et}}(), \\
\bar{\varphi}_{*}: K_{*}\left(, \mathbf{Z} / l^{\nu}\right) \rightarrow K_{*}^{\mathrm{et}}\left(, \mathbf{Z} / l^{\nu}\right), \quad l^{\nu} \neq 2,
\end{gathered}
$$

defined on the category of noetherian $\mathrm{Z}[1 / l]$-algebras. For any finite, étale extension $A \rightarrow A^{\prime}$ of noetherian $\mathrm{Z}[1 / l]$ algebras, $\hat{\varphi}_{*}$ and $\bar{\varphi}_{*}$ commute with the transfer maps on algebraic and étale $K$-theory.

There are relationships between étale $K$-theory and étale cohomology given by spectral sequences of Atiyah-Hirzebruch type.

Proposition 5. For any noetherian $\mathrm{Z}[1 / l]$-algebra $A$ of finite mod $l$ étale cohomological dimension, there exist natural "fringed" spectral sequences

$$
\begin{gathered}
E_{2}^{p, q}=H^{p}\left(A, \mathrm{Z}_{l}(-q / 2)\right) \Rightarrow \hat{K}_{-p-q}^{\mathrm{et}}(A), \\
E_{2}^{p, q}=H^{p}\left(A, \mathrm{Z} / l^{\nu}(-q / 2)\right) \Rightarrow K_{-p-q}^{\mathrm{et}}\left(A, \mathrm{Z} / l^{\nu}\right)
\end{gathered}
$$

where $\mathbf{Z}_{l}(-q / 2)=0=\mathbf{Z} / l^{\nu}(-q / 2)$ unless $q$ is a nonpositive even integer.

These spectral sequences necessarily degenerate for rings $A$ of $\mathbf{Z} / l$ cohomological dimension at most 2 (e.g. $O[1 / l]$ in Theorem 1 or $A$ in Theorem 3). In view of this, Theorems 1 and 3 are implied by the following theorem.

THEOREM 6. Let $A$ denote either a ring of integers in a function field of characteristic $p \neq l$, or $O[1 / l]$ with $O$ a ring of integers in a number field. In the 
second case, assume that the quotient field of $A$ is totally imaginary if $l=2$. Then for any $v>0(v>1$ if $l=2)$ the natural maps

$$
\bar{\varphi}_{*}: K_{j}\left(A, \mathbf{Z} / l^{\nu}\right) \rightarrow K_{j}^{\mathrm{et}}\left(A, \mathbf{Z} / l^{\nu}\right) \quad(j>1)
$$

are surjective.

In the case in which $A$ contains a primitive $l^{v}$ th root of unity (denoted $\zeta_{l} v$ ) the proof of Theorem 6 is not difficult because $K_{*}^{\text {et }}\left(A, \mathrm{Z} / l^{\nu}\right)$ is then periodic of period 2. To prove Theorem 6 in general, we combine this surjectivity of $\bar{\varphi}_{*}$ for $A^{\prime}=A\left[\zeta_{l} v\right]$ with the following secondary transfer theorem.

Theorem 7. Let $A, l, v$ be as in Theorem 6 , let $A^{\prime}=A\left[\zeta_{l} v\right]$, and let $T \in$ $\operatorname{Gal}\left(A^{\prime}, A\right)$ be a generator. Then for any $i>0$ there exists a natural commutative square

$$
\operatorname{Ker}\left\{K_{i}\left(A^{\prime}, \mathrm{Z} / l^{\nu}\right) \stackrel{1-T}{\longrightarrow} K_{i}\left(A^{\prime}, \mathrm{Z} / l^{\nu}\right)\right\} \rightarrow \operatorname{coker}\left\{K_{i+1}\left(A^{\prime}, \mathrm{Z} / l^{\nu}\right) \stackrel{\text { tr }}{\rightarrow} K_{i+1}\left(A, \mathrm{Z} / l^{\nu}\right)\right\}
$$

$\operatorname{Ker}\left\{K_{i}^{\mathrm{et}}\left(A^{\prime}, \mathrm{Z} / l^{\nu}\right) \stackrel{1-T}{\longrightarrow} K_{i}^{\mathrm{et}}\left(A^{\prime}, \mathrm{Z} / l^{\nu}\right)\right\} \rightarrow \operatorname{coker}\left\{K_{i+1}^{\mathrm{et}}\left(A^{\prime}, \mathrm{Z} / l^{\nu}\right) \stackrel{\mathrm{tr}}{\rightarrow} K_{i+1}^{\mathrm{et}}\left(A, \mathrm{Z} / l^{\nu}\right)\right\}$

whose lower horizontal arrow is surjective, where tr denotes the transfer map.

\section{BIBLIOGRAPHY}

1. A. Borel, Stable real cohomology of arithmetic groups, Ann. Sci. École Norm. Sup. (4) 7 (1972), 235-272.

2. W. Dwyer, E. Friedlander, V. Snaith and R. Thomason, Algebraic K-theory eventually surjects onto topological $K$-theory (preprint).

3. E. M. Friedlander, Etale $K$-theory. II: Connections with algebraic $K$-theory, Ann. Sci. École Norm. Sup. (4) (to appear).

4. B. Harris and G. Segal, $K_{i}$ of rings of algebraic integers, Ann. of Math. (2) 101 (1975), 20-33.

5. S. Lichtenbaum, Values of zeta functions, étale cohomology and algebraic $K$-theory, Lecture Notes in Math., vol. 342, Springer-Verlag, Berlin and New York, 1973.

6. C. Soulé, $K$-theorie des anneaux d'entiers de corps de nombres et cohomologie étale, Invent. Math. 55 (1979), 251-295.

7. - On higher p-adic regulators, Lecture Notes in Math., vol. 854, SpringerVerlag, Berlin and New York, 1980.

8. R. Thomason, Algebraic K-theory and étale cohomology (preprint).

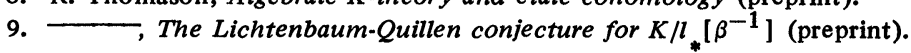

DEPARTMENT OF MATHEMATICS, UNIVERSITY OF NOTRE DAME, P. O. BOX 398, NOTRE DAME, INDIANA 46556 (Current address of W. G. Dwyer)

DEPARTMENT OF MATHEMATICS, NORTHWESTERN UNIVERSITY, EVANSTON, ILLINOIS 60201 (Current address of E. M. Friedlander) 